5. Gunson, J.: Structure of quantum mechanics. Commun. math. Phys. 6, 262-285 (1967).

6. Haag, R., Kastler, D. : An algebraic approach to quantum field theory. J. Math. Phys. $5,848-861$ (1964).

7. Jauch, J.: Foundations of quantum mechanics. Reading, Mass.: Addison-Wesley 1968.

8. Jauch, J., Misra, B., Gibson, A.: On the asymptotic condition of scattering theory. Helv. Phys. Acta 41, 513-527 (1968).

9. Mielnik, B.: Geometry of quantum states. Commun. math. Phys. 9, 55-80 (1968).

10. Mielnik, B.: Theory of filters. Commun. math. Phys. 15, 1-46 (1969).

11. Neumann, H.: Classical systems and observables in quantum mechanics. Commun. math. Phys. 23, 100-116 (1971).

12. Peressini, A.: Ordered Topological Vector Spaces. New York: Harper and Row 1967.

13. Stolz, P.: Attempt of an axiomatic foundation of quantum mechanics and more general theories, VI. Commun. math. Phys. 23, 117-126 (1971).

14. Segal, I.: Mathematical problems of relativistic physics. American Math. Soc. Lectures in Applied Mathematics. Providence, R.I. (1963).

15. Varadarajan, V.: Geometry of Quantum Theory, Vol. I. Princeton, N.J.: Van Nostrand 1968.

S. Gudder

Department of Mathematics

University of Denver

Denver Colorado 80210, USA

\title{
Erratum
}

\section{Non-Existence of Axially Symmetric Massive Scalar Fields}

\author{
A. R. Roy and J. R. Rao \\ Commun. math. Phys. 27, 162-166 (1972)
}

Page 165: 4th line onwards from the top reads as follows:

Which in view of (3.4) implies

$$
-\mu^{2} V^{2}+g^{11} g^{44}\left(F_{14}\right)^{2}-g^{22} g^{33}\left(F_{23}\right)^{2}=0 .
$$

Since $g^{14}, g^{22}, g^{33}$ are all negative and $g^{44}$ is positive, (3.5) will hold iff

$$
\mu=0, \quad F_{14}=0, \quad F_{23}=0 .
$$

Hence, there cannot exist any solution for the coupled electromagnetic and massive scalar fields for the metric (2.1).

The rest of the calculations are unnecessary and may, therefore, be ignored. 